\title{
Multi-vortex laser enabling spatial and temporal encoding
}

\author{
Zhen Qiao ${ }^{1 \dagger}$, Zhenyu Wan ${ }^{2 \dagger}$, Guogiang Xie ${ }^{1 *}$, Jian Wang ${ }^{2 *}$, Liejia Qian ${ }^{1}$ and Dianyuan Fan ${ }^{1,3}$
}

\author{
* Correspondence: xiegq@sjtu.edu. \\ cn; jwang@hust.edu.cn \\ 'Zhen Qiao and Zhenyu Wan \\ contributed equally to this work. \\ ${ }^{1}$ School of Physics and Astronomy, \\ Key Laboratory for Laser Plasmas \\ (Ministry of Education), \\ Collaborative Innovation center of \\ IFSA (CICIFSA), Shanghai Jiao Tong \\ University, Shanghai 200240, China \\ ${ }^{2}$ Wuhan National Laboratory for \\ Optoelectronics and School of \\ Optical and Electronic Information, \\ Huazhong University of Science and \\ Technology, Wuhan 430074, China \\ Full list of author information is \\ available at the end of the article
}

\begin{abstract}
Optical vortex is a promising candidate for capacity scaling in next-generation optical communications. The generation of multi-vortex beams is of great importance for vortex-based optical communications. Traditional approaches for generating multivortex beams are passive, unscalable and cumbersome. Here, we propose and demonstrate a multi-vortex laser, an active approach for creating multi-vortex beams directly at the source. By printing a specially-designed concentric-rings pattern on the cavity mirror, multi-vortex beams are generated directly from the laser. Spatially, the generated multi-vortex beams are decomposable and coaxial. Temporally, the multi-vortex beams can be simultaneously self-mode-locked, and each vortex component carries pulses with $\mathrm{GHz}$-level repetition rate. Utilizing these distinct spatial-temporal characteristics, we demonstrate that the multi-vortex laser can be spatially and temporally encoded for data transmission, showing the potential of the developed multi-vortex laser in optical communications. The demonstrations may open up new perspectives for diverse applications enabled by the multi-vortex laser.

Keywords: Multi-vortex laser, Spatial encoding, Temporal encoding
\end{abstract}

\section{Introduction}

Optical vortices, having distinct features of helical phase front and doughnut intensity profile, have attracted increasing interests in a variety of applications such as optical manipulation, trapping, tweezers, microscopy, imaging, metrology, nonlinear interactions, astronomy, and quantum science in the past decades [1-10]. Recently, optical vortices, which provide in principle unlimited number of orbital angular momentum (OAM) quantum states [11], are used as powerful tools in the emerging application of optical communications [12-29]. By applying multiple vortex beams with various topological charges, the communication capacity will be further increased beyond the traditional well-established physical dimensions such as wavelength and polarization [15, 16, 25, 26]. Moreover, vortex-based data transmission can also potentially enhance the communication security $[12,22]$. Contributed by these advantages, optical vortex becomes a promising candidate for capacity scaling in next-generation optical communications.

The generation of multi-vortex beams is the prerequisite of vortex-enabled optical communications. Up to now, due to lack of a multi-vortex laser source, it has always been relying on spatially modulating Gaussian beams through multiple spatial phase

(c) The Author(s). 2020 Open Access This article is licensed under a Creative Commons Attribution 4.0 International License, which permits use, sharing, adaptation, distribution and reproduction in any medium or format, as long as you give appropriate credit to the original author(s) and the source, provide a link to the Creative Commons licence, and indicate if changes were made. The images or other third party material in this article are included in the article's Creative Commons licence, unless indicated otherwise in a credit line to the material. If material is not included in the article's Creative Commons licence and your intended use is not permitted by statutory regulation or exceeds the permitted use, you will need to obtain permission directly from the copyright holder. To view a copy of this licence, visit http://creativecommons.org/licenses/by/4.0/. 
elements, such as spatial light modulators (SLM) [30] and spiral phase plates (SPP) [31], to generate multi-vortex beams for data transmission. In this way, additional cumbersome beam splitters are always used for combing multiple vortex beams together, resulting in an unscalable and bulky communication system. Although the superposition of multi-vortex beams can be created with a single SLM by loading a specific complex phase pattern [32,33], the quality of the generated superimposed vortex beams is relatively poor due to the limited resolution of the commercially available SLMs, especially for high-order OAM-carrying optical vortices with fine spatial structures. Therefore, traditional techniques of generating multi-vortex beams limit the applications of high-order vortex-based optical communications. Meanwhile, most of the previous demonstrations on multi-vortex beams are based on passive techniques. In this scenario, it is worthwhile developing novel active techniques of creating multivortex beams directly at the source.

Because Laguerre-Gaussian (LG) vortex beams are eigen transverse modes of laser oscillation in cavity, vortex beams can be generated directly from laser. In principle, the vortex beams generated from laser possess pure spiral phases and are stable for transmission. Such high-quality beam generation will benefit high-performance vortex-based data transmission, e.g. in the aspect of long-distance transmission. However, attempts have always been made to generate vortex beams with only single topological charge [34-38]. Different from single vortex generation, generation of multi-vortex beams from laser requires finely controlling of transverse modes in sub-mode scale and breaking the competitions among transverse modes, which has not been realized so far. Therefore, it is quite a challenge to generate multi-vortex beams directly from laser.

In this article, we present creation of multi-vortex beams at the laser source by printing a specially-designed concentric-rings pattern on cavity mirror. In theory, we propose multi-vortex oscillation threshold minimum analyzing (MOTMA) method to design the pattern printed on cavity mirror that supports multi-vortex beams generation. In the experiment, a concentric-rings pattern designed using MOTMA method is printed on the surface of laser output coupler $(\mathrm{OC})$, and multi-vortex beams with various topological charges are simultaneously generated from laser. Spatially, the generated multi-vortex beams are decomposable and coaxial. Temporally, the multi-vortex beams can be simultaneously self-mode-locked, and each vortex component carries pulses with GHz-level repetition rate. Applying these unique spatial-temporal characteristics, we demonstrate that the multi-vortex laser can be spatially and temporally encoded for data transmission, which shows the potential of the developed multivortex laser to be applied in optical communications.

\section{Results}

\section{Concept and principle}

The concept and principle of multi-vortex beams generation from laser for spatial and temporal encoding is schematically illustrated in Fig. 1. Determined by the intensity distribution of $\mathrm{LG}_{0}$ vortex beam ( $l$ is the topological charge), the bright ring radius of optical vortex increases with the topological charge number $l$. Therefore, multi-vortex beams that are spatially separated can be concentrically constructed by finely controlling transverse modes. By spatially separating different vortex modes in gain medium, 


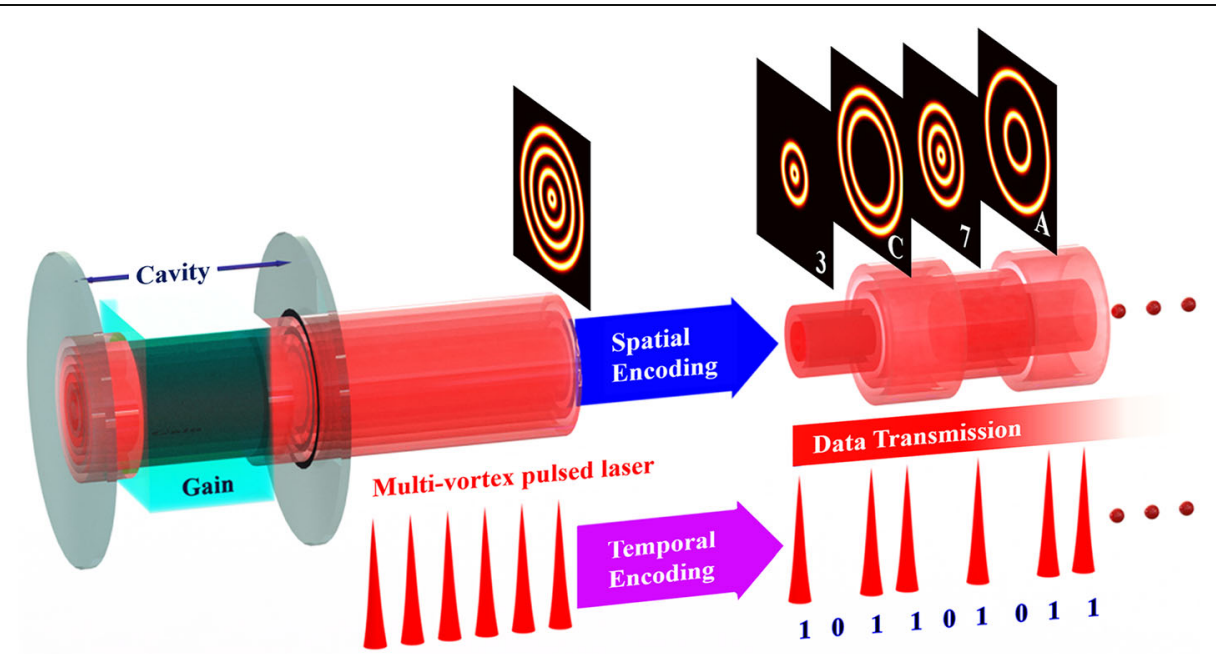

Fig. 1 Concept and principle. Multi-vortex pulsed beams are generated directly from the laser. Spatial encoding is achieved by combining specific components of the vortex beams, and temporal encoding is achieved by modulating the pulses. Both spatial and temporal encoding are used for data transmission

gain competitions among the vortex modes are avoided, thus multi-vortex beams can steadily oscillate and output from laser. Moreover, each vortex component can carry high-repetition pulses through self-mode locking operation of the multi-vortex laser. By applying the unique spatial and temporal dimensions provided by the multi-vortex laser, spatial and temporal encoding can be performed. On one hand, spatial encoding can be achieved by arbitrarily combining the vortex components as spatial codes. On the other hand, temporal encoding can be achieved by modulating the mode-locked pulses as temporal codes. Both spatial and temporal encoding can be applied for data transmission.

\section{Design of multi-vortex laser}

For generating multi-vortex beams, an end-pumped laser with a cylindrically symmetric cavity structure is designed, as schematically illustrated in Fig. 2a. The laser cavity is composed of a concave mirror M1 and a plano-plano output coupler (OC) with a gain crystal. The pumping light emitted from a laser diode (LD) is collimated by lens L1, focused by lens L2, and then is incident into the gain crystal. In particular, for constructing multi-vortex beams to oscillate, we propose to design a specific pattern and print it on the surface of OC to form loss modulations. In order to obtain the pattern that supports the generation of multi-vortex beams with specific topological charges, multivortex oscillation threshold minimum analyzing (MOTMA) method is proposed. In laser, those vortices having oscillation threshold minima will be selected to oscillate, thus we only need to find a pattern for providing loss modulations so that the objective vortices have the oscillation threshold minima. It is assumed that the pattern is composed of a solid circle in the center and multiple concentric rings (Fig. 2c). When the center of this concentric-rings pattern is positioned on the laser axis, loss modulations will be introduced and it will form multiple ring-shaped laser passes in the unprinted region which match with the objective multi-vortex beams. The concentric-rings 


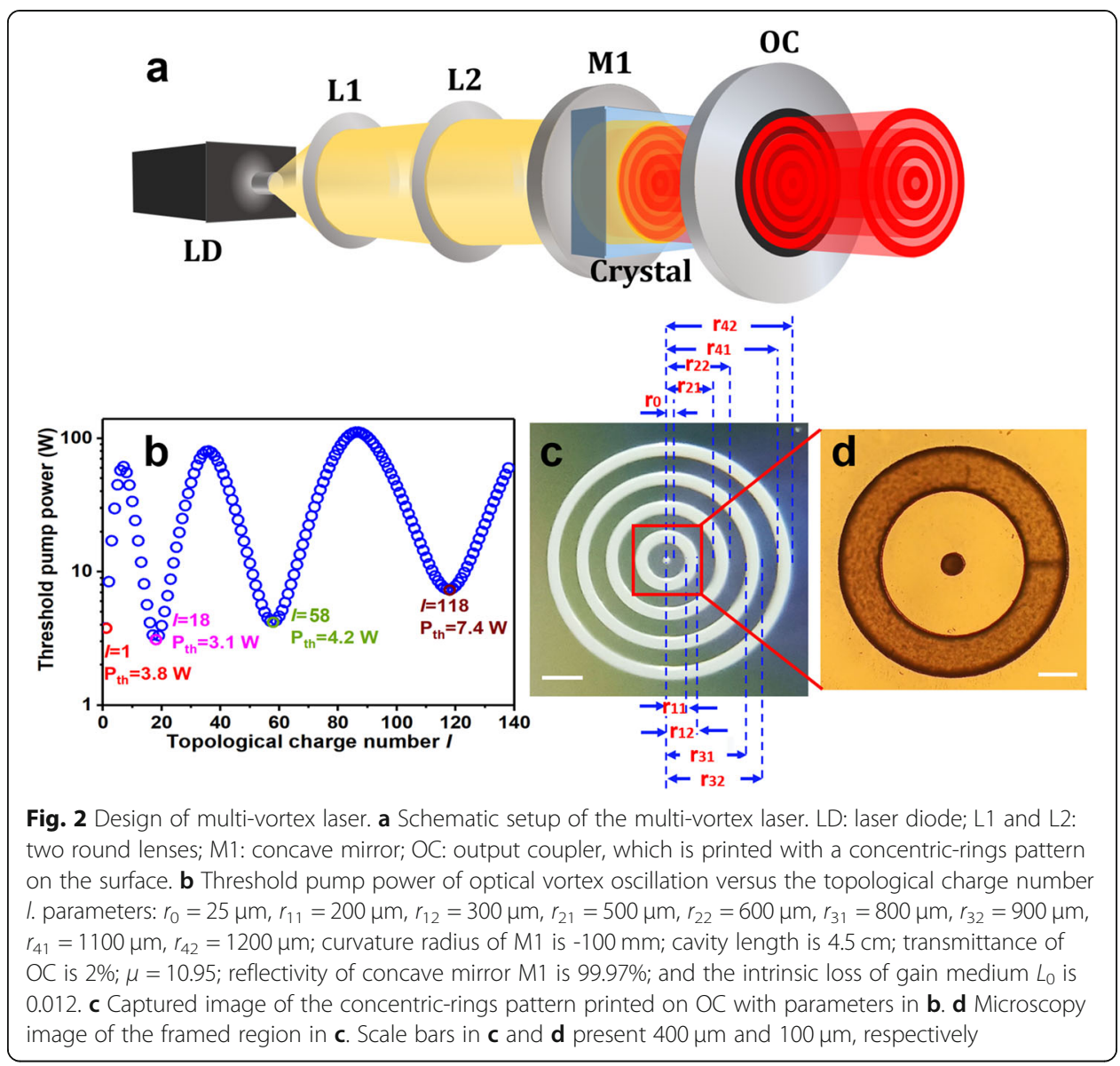

pattern can be completely described by the radius of the central circle $r_{0}$, the inside radius of the $i$-th ring $r_{i 1}$, the outside radius of the $i$-th ring $r_{i 2}$, and the number of the rings $n$.

As an example, we consider the generation of four vortex beams with topological charges of $l=1, l=18, l=58$, and $l=118$, and the oscillation pump thresholds of these four vortex beams should be the minima. Based on such consideration, the oscillation pump threshold versus the topological charge number $l$ of $\mathrm{LG}_{0 l}$ vortex mode is calculated by taking a specific concentric-rings pattern printed on OC into account (For details refer to Methods and Supplementary Information Section 1). As shown in Fig. 2b, it presents four minima at $l=1, l=18, l=58$ and $l=118$. In this case, parameters of the concentric-rings pattern are designed to be $r_{0}=25 \mu \mathrm{m}, r_{11}=200 \mu \mathrm{m}, r_{12}=300 \mu \mathrm{m}, r_{21}=$ $500 \mu \mathrm{m}, r_{22}=600 \mu \mathrm{m}, r_{31}=800 \mu \mathrm{m}, r_{32}=900 \mu \mathrm{m}, r_{41}=1100 \mu \mathrm{m}, r_{42}=1200 \mu \mathrm{m}$, and the concentric-rings pattern is depicted in Fig. 2c. In fact, because the four vortex beams are spatially separated and there are no gain competitions among the vortex beams, these four optical vortex beams will be selected to simultaneously oscillate and output from the laser when the pump power is above all the four threshold minima. With MOTMA method, quantitative relationship between the concentric-rings pattern and the topological charge numbers of the multi-vortex beams can be obtained. Therefore, multi-vortex beams with specific topological charges can be controllably generated from the laser by specially designing the concentric-rings pattern printed on OC with 
MOTMA method. This method for multi-vortex beams generation is reliable and versatile.

\section{Experiment of multi-vortex generation}

In the experiment, a multi-vortex laser with a $\mathrm{Nd}$ : $\mathrm{YVO}_{4}$ crystal as the gain medium is built as designed in Fig. 2a (For details refer to Methods). The pump beam has a near Gaussian intensity distribution (inset of Fig. 3k). For generating multi-vortex beams, a concentricrings pattern with $r_{0}=25 \mu \mathrm{m}, r_{11}=200 \mu \mathrm{m}, r_{12}=300 \mu \mathrm{m}, r_{21}=500 \mu \mathrm{m}, r_{22}=600 \mu \mathrm{m}, r_{31}=$ $800 \mu \mathrm{m}, r_{32}=900 \mu \mathrm{m}, r_{41}=1100 \mu \mathrm{m}$ and $r_{42}=1200 \mu \mathrm{m}$ is printed on the surface of OC, which is shown in Fig. 2c (These parameters are the same as those designed). We adjust the center of the concentric-rings pattern to the cavity axis. As expected, multi-vortex beams are simultaneously generated from the laser. The measured intensity profile of the generated multi-vortex beams is shown in Fig. 3a, which is recorded by a CCD camera. It presents four typical doughnut shapes distributed concentrically. The measured intensity profiles of the four vortex components are shown in Fig. 3b-e, respectively. The topological charges of the generated multi-vortex beams are measured by converting the $\mathrm{LG}_{0 l}$ vortex modes into the corresponding $\mathrm{HG}_{0 l}$ modes using a cylindrical lens mode converter $[39,40]$. The nodal lines number in the $\mathrm{HG}_{0 l}$ mode profile is equal to the topological charge number of the vortex. In the experiment, apertures are used to separately select the multi-vortex components to pass through the cylindrical lens mode converter, and Hermite-Gaussian (HG) beams converted from the multi-vortex components are obtained as shown in Fig. 3g-j. By counting the nodal lines of the HG beams, the topological charges of the generated multi-vortex beams are measured to be $l=1, l=18, l=58$, and $l=118$, respectively. The topological charges of the generated multi-vortex beams are perfectly consistent with the theoretical design results obtained with MOTMA method. Therefore, it further presents the reliability of the MOTMA method. The output powers of discrete vortex beams versus the incident pump power are shown in Fig. 3k. The measured threshold pump powers of the discrete vortex beams are 4.0 W, 3.7 W, 4.8 W and 8.0 W for $\mathrm{LG}_{01}, \mathrm{LG}_{0,18}, \mathrm{LG}_{0,58}$, and $\mathrm{LG}_{0,118}$ vortices, respectively, which are in good agreement with the calculated threshold pump powers

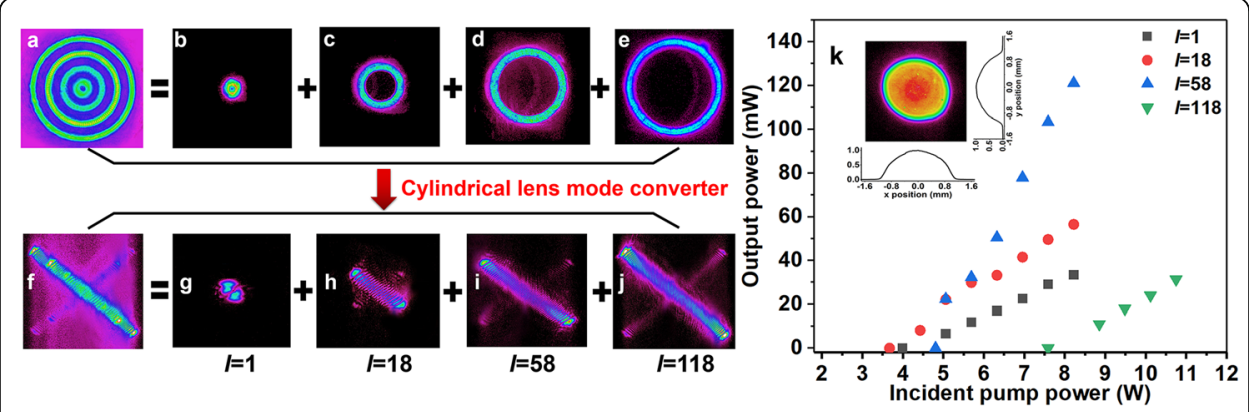

Fig. 3 Multi-vortex beams generation. a Measured intensity profile of the generated multi-vortex beams. $\mathbf{b}$, $\mathbf{c}, \mathbf{d}$, e Measured intensity profiles of the vortex components of the multi-vortex beams. $\mathbf{f}$, Intensity profile of the multi-HG beams converted from the generated multi-vortex beams by a cylindrical lens mode converter. $\mathbf{g}, \mathbf{h}, \mathbf{i}, \mathbf{j}$ Intensity profiles of the HG beams converted from the vortex components, showing that the topological charges of the multi-vortex beams are $I=1, I=18, I=58$, and $I=118$, respectively. $\mathbf{k}$ Output powers of the discrete vortex beams versus the incident pump power; Inset: pump beam profile 
of $3.8 \mathrm{~W}, 3.1 \mathrm{~W}, 4.2 \mathrm{~W}$ and 7.4 W for $\mathrm{LG}_{01}, \mathrm{LG}_{0,18}, \mathrm{LG}_{0,58}$, and $\mathrm{LG}_{0,118}$ vortices shown in Fig. 2b (Details refer to Methods and Supplementary Information Section 1).

Some experimental evidences suggest that self-mode-locking operation of high-order transverse mode with high-repetition pulses can be achieved in short-cavity lasers [41-44]. Actually, high-repetition pulses with $\mathrm{GHz}$ level are ideal sources for temporal encoding and time-division multiplexing (TDM) in optical communications [45, 46]. This also motivates us to study the temporal characteristics of the generated multi-vortex beams. Considering that the multi-vortex laser possesses a short cavity cycle of about $300 \mathrm{ps}(\sim 3.2 \mathrm{GHz}$ in repetition rate), a high-speed photodetector and a high-speed oscilloscope are used to detect and record the temporal characteristics. As expected, we find that the multi-vortex beams can be simultaneously self-mode-locked by finely optimizing the cavity. As shown in Fig. 4, the self-mode-locked vortex beams all possess stable pulse trains. By measuring the radio frequency (RF) spectrum of the mode-locked pulses, signal-to-noise ratio of over $40 \mathrm{~dB}$ and high pulse repetition of $3.2 \mathrm{GHz}$ are presented. Besides, the pulse widths of the modelocked multi-vortex beams are 48 ps. The detailed mode locking results can be referred to Supplementary Information Section 2. In fact, self-mode locking is a general phenomenon in narrow-band lasers $[43,44,47,48]$. Due to Stark splitting of gain medium induced by intracavity laser field, there will be a dip in the gain-line shape. The laser with such a concave gain shape will produce short pulses due to the pulse stabilization mechanism where spectrum broadening or narrowing will both cause lower average gain [49]. It is worth mentioning that, Nd: YVO4 crystal possesses a large nonlinear refractive index, so the combination of Kerr-lens and hard-aperture of the printed concentric-ring pattern on OC may also contribute to the self-mode locking operation.

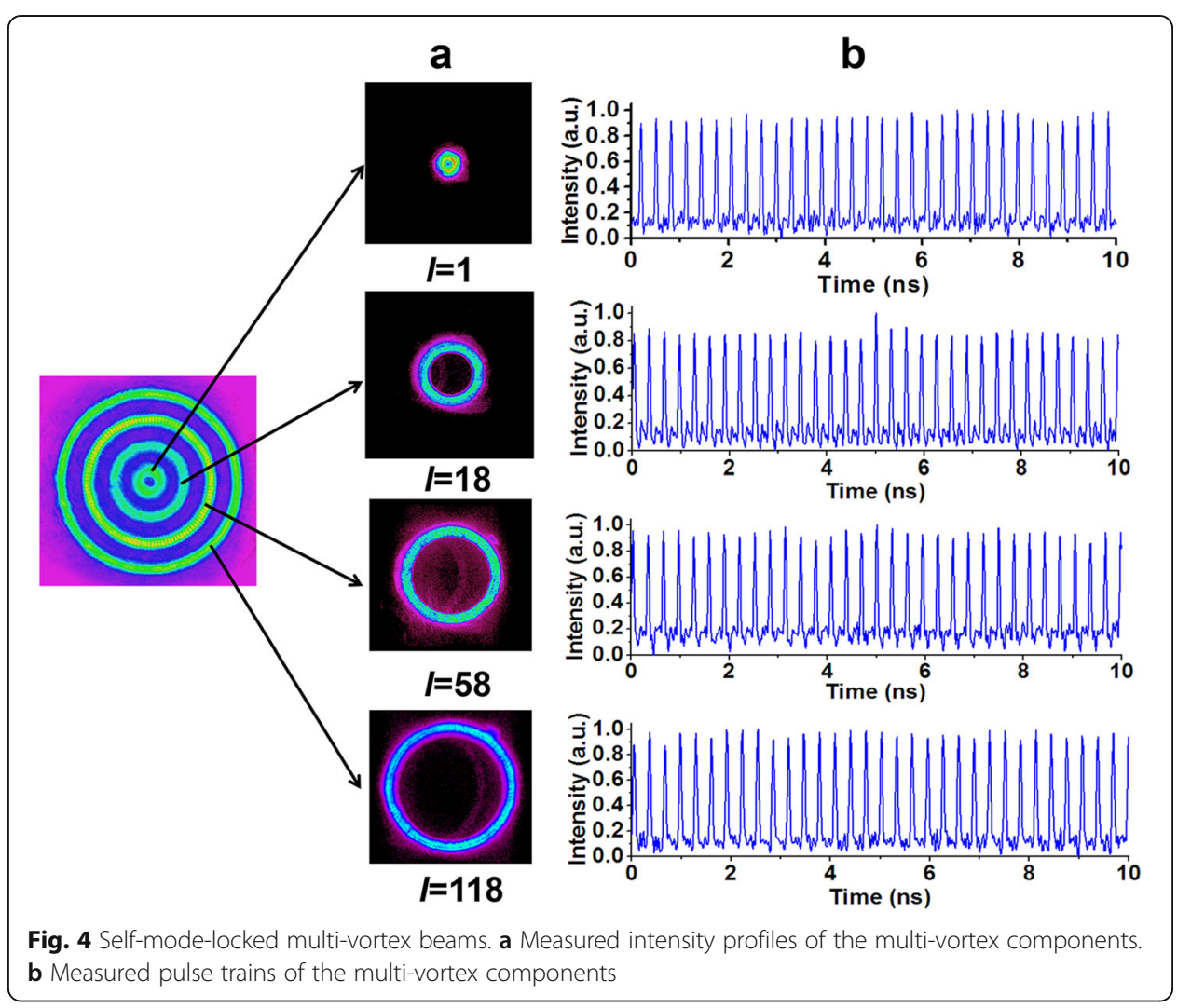


Remarkably, the obtained results show that the generated multi-vortex beams are naturally decomposable and coaxial. Moreover, the multi-vortex beams carry highrepetition picosecond pulses. Therefore, compared with traditional single-charge continuous wave $(\mathrm{CW})$ vortex lasers, the developed multi-vortex laser increases two available spatial and temporal dimensions, which provides favorable potential to perform spatial and temporal encoding for data transmission.

\section{Spatial encoding of the multi-vortex laser}

For showing the potential of the multi-vortex laser in optical communications, proofof-concept experiments are performed. Here, spatial encoding and data transmission are demonstrated in the experiment. Considering that the number of the generated multi-vortex beams is four, hexadecimal encoding of the generated vortex beams is proposed. We define "none of beams" as " 0 ", the first vortex beam $(l=1)$ as " $20=1$ ", the second vortex beam $(l=18)$ as " $2^{1}=2$ ", the third vortex beam $(l=58)$ as " $2^{2}=4$ ", and the fourth vortex beam $(l=118)$ as " $2^{3}=8$ ". Then the complete hexadecimal encoding can be achieved by selectively combining the multi-vortex components. For example, "D" in hexadecimal codes represents "13" in decimal codes. Given that " $13=$ $1+4+8$ ", the combination of the first, the third and the fourth vortex beams can be encoded as " $D$ " in hexadecimal codes.

To demonstrate the spatial encoding and free-space data transmission, we build up a data transmission system as shown in Fig. 5a. First, a digital micromirror device (DMD) is used to encode the multi-vortex laser by controlling specific combinations of the vortex components to be reflected for data transmission. Then, the encoded multi-vortex
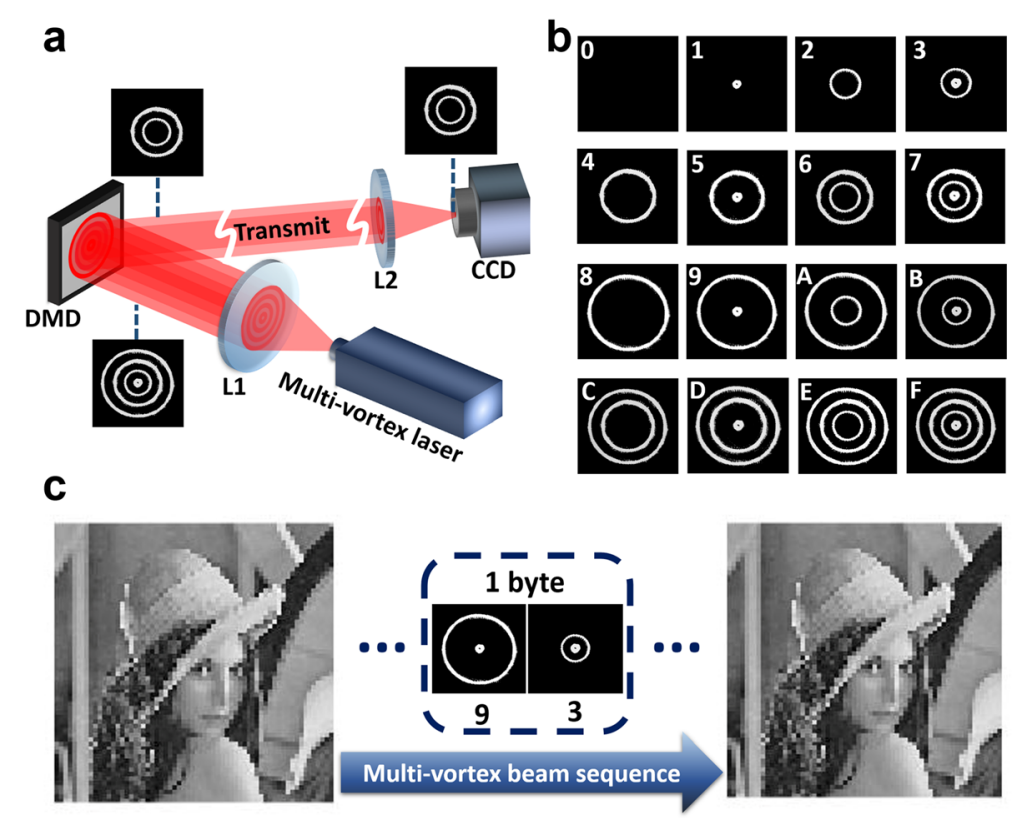

Fig. 5 Spatial encoding of the multi-vortex laser for data transmission. a Schematic of data transmission by spatial encoding of the multi-vortex laser. DMD: digital micromirror device. $\mathbf{b}$ Measured patterns of the multi-vortex laser encoded by DMD and the corresponding hexadecimal codes. c Measured data transmission results for image transfer using spatial encoding of the multi-vortex laser 
laser transmits in free space. Finally, a lens is used to focus the multi-vortex beam into a CCD camera, and the data is decoded according to the recorded multi-vortex patterns. The measured patterns of the encoded multi-vortex laser and the corresponding hexadecimal codes are shown in Fig. 5b. By following the above process, a grayscale image with $64 \times 64$ pixels is transmitted using the built data transmission system. In a grayscale image, each pixel possesses one of $256 \mathrm{~Gy}$ scales. Because $256=16 \times 16$, the grayscale value of each pixel is encoded by two hexadecimal sequences. As shown in Fig. $5 \mathrm{c}$, in the data transmission experiment, there is no errors for the transmitted image [bit error rate (BER) is zero], showing the feasibility of data transmission by applying spatial encoding of the multi-vortex laser.

\section{Temporal encoding and time-division multiplexing}

The above spatial encoding utilizes the spatial dimension of the multi-vortex laser. Actually, ultrafast optical vortices with high-repetition pulses can be applied for timedivision multiplexing (TDM) [50], which can further increase the communication capacity. Here, by applying the generated picosecond-pulse multi-vortex beams, temporal encoding and TDM are experimentally demonstrated. The process of TDM of the picosecond vortex is shown in Fig. 6a. In the experiment, the pulsed $L G_{01}$ vortex beam is employed. First, the pulsed $\mathrm{LG}_{01}$ vortex beam is divided into two pulsed beams. Then, the two pulsed beams are separately temporally encoded with binary signals by pulse

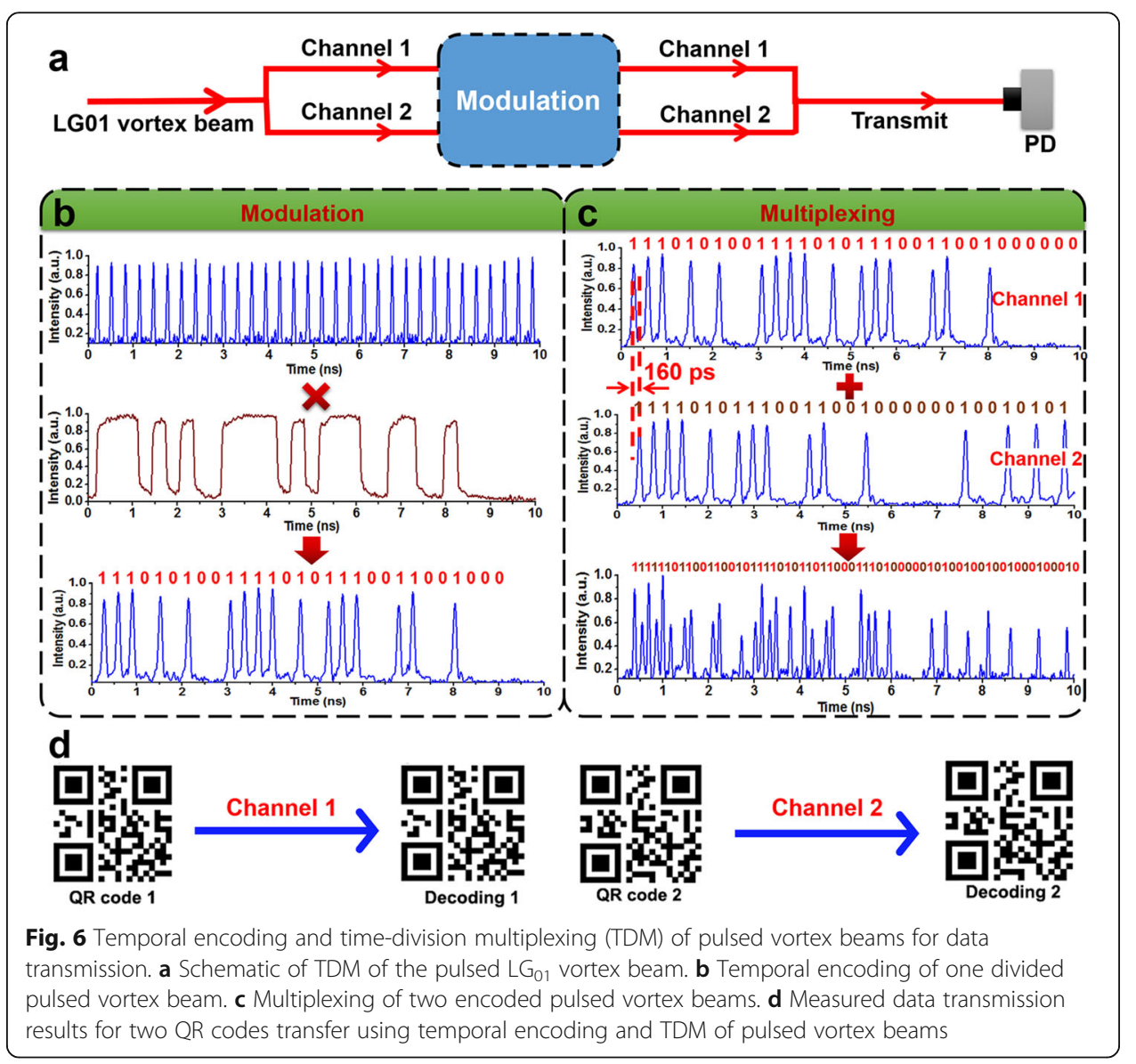


modulation. Finally, the two encoded beams are multiplexed for data transmission. At the receiver end, the multiplexed beams are received by a photodetector and decoded by computer.

In the process of temporal encoding, as shown in Fig. 6b, the pulsed beam is modulated by an electro-optic modulator whose modulation function is shown in the middle part of Fig. 6b. After the modulation, the pulsed vortex beam carries binary signals as shown in the bottom part of Fig. $6 \mathrm{~b}$, where the "pulse" moments represent " 1 " and the "non-pulse" moments represent " 0 ". With the same modulation process, another divided pulsed beam is also temporally encoded. Multiplexing of the two encoded pulsed beams is achieved by combining them with a half-pulse-cycle delay (Fig. 6c). The multiplexed pulse trains, as shown in the bottom part of Fig. 6c, carry two groups of binary signals. For demonstrating the feasibility of TDM of the pulsed vortex beam, two different QR codes are used for temporal-encoding data transmission. During the temporal encoding, the black pixels of QR codes are encoded as "1" and the white pixels of QR codes are encoded as "0". As shown in Fig. 6d, two QR codes are successfully transmitted with a BER of zero. Therefore, we demonstrate that the pulsed vortex beams can be temporally encoded and applied in TDM. Each of the optical vortices can be applied for data transmission through TDM, and the TDM traditionally with Gaussian beam can be extended to vortex beam. The multi-vortex beams enable not only TDM but also SDM, thus increasing a new dimension of space for data transmission comparing with the traditional Gaussian beam-based data transmission. The proof-of-concept experiment for simultaneously time-space division multiplexing (TSDM) of multi-vortex beams is also performed, as shown in Supplementary Information Section 3. It is worth mentioning that, mode-locking pulses with higher repetition rate can be obtained (even $100 \mathrm{GHz}$ level can be achieved [51]) when further decreasing the cavity length of the multi-vortex laser, and the data transmission rate will be further increased.

The obtained results shown in Figs. 5 and 6 indicate the successful implementation of the multi-vortex laser for spatial and temporal encoding data transmission.

\section{Discussion}

We demonstrate that the multi-vortex laser is directly encoded in the spatial dimension. From another point of view, the performed hexadecimal encoding of the four vortex beams in the experiment is equivalent to that each vortex component carries different binary codes. The performed spatial encoding is analogous to the concept of space-division multiplexing (SDM) of multi-vortex beams. In principle, a free-spacetype modulator, which is able to modulate light beams in local space, can be utilized to load independent signals onto each vortex component of the multi-vortex beams, and direct multiplexing of the multi-vortex beams will be achievable. Because the multivortex beams are naturally coaxial with transmission determined by the cylindrical symmetry of the laser cavity, such direct multiplexing will significantly save the unscalable and bulky systems required in traditional vortex-based data transmission, which shows the potential of the multi-vortex laser to be applied in vortex-enabled capacity scaling of optical communications. Actually, by specially designing the concentric-rings pattern printed on the cavity mirror using the proposed MOTMA method, vortex beams with higher order and more topological charges can be generated, which will further increase the communication capacity. This can significantly expand the 
available high-order optical vortices in optical communications, which are far beyond most of the previous demonstrations of vortex-based communications only employing low-order optical vortices [12-26].

\section{Conclusions}

In conclusion, multi-vortex beams are simultaneously generated from laser by printing a specially-designed concentric-rings pattern on the cavity mirror. In theory, MOTMA method is proposed to obtain the relationship between the concentric-rings pattern parameters and the topological charges of multi-vortex beams, which provides a reliable and versatile design method for direct multi-vortex beams generation from laser. In the experiment, by printing the specially-designed concentric-rings pattern on the OC, multi-vortex beams with four topological charges are simultaneously generated, and the results are perfectly consistent with the theoretical design results obtained with the MOTMA method. Through self-mode-locking operation, pulses with GHz-level repetition rate are carried by the multi-vortex beams. By applying the developed multi-vortex laser, spatial encoding and free-space data transmission are demonstrated. Moreover, temporal encoding and TDM are also demonstrated by using the mode-locked pulses. Therefore, the developed multi-vortex laser provides the added spatial and temporal dimensions that can be encoded, and has a great potential to be applied in optical communications for capacity scaling. With future improvement, the developed multi-vortex laser with distinct spatial-temporal characteristics may also find other emerging applications in manipulation, trapping, microscopy, metrology and material processing with superior performance.

\section{Methods}

\section{Derivation of relationship between the pump threshold and the topological charge of} vortex

When using MOTMA method to design specific multi-vortex beams generation, relationship between the pump thresholds and the topological charge numbers should be first obtained. Here, the process is conducted. Now consider $\mathrm{LG}_{0 l}$ vortex mode oscillating in cavity, whose pump threshold is proportional to the cavity loss and is inversely proportional to the laser gain. Thus, the threshold pump power for $\mathrm{LG}_{0 l}$ mode oscillation can be expressed as [52].

$$
P_{t h}\left(L G_{0 l}\right) \propto \frac{L_{0}+L_{l}\left(T_{0}, r_{0}, r_{i 1}, r_{i 2}, n, R\right)}{\int r_{p}(r, z) s_{0 l}(r, z) d V}
$$

where $r_{p}(r, z)$ and $s_{0 l}(r, z)$ are the normalized pump intensity distribution and normalized laser intensity distribution, respectively. The integral represents the laser gain. $L_{0}$ is the loss of the gain medium. $L_{l}$ is the loss of cavity mirrors for $\mathrm{LG}_{0 l}$ mode, and it is related to the $\mathrm{OC}$ transmittance $\left(T_{0}\right)$, the concentric-rings pattern parameters $r_{0}, r_{i 1}, r_{i 2}$, the number of the concentric rings $n$, and the reflectivity $R$ of the concave mirror (M1 in Fig. 2a). $L_{l}$ can be expressed as (For details refer to Supplementary Discussion I) 


$$
L_{l}\left(T_{0}, r_{0}, r_{i 1}, r_{i 2}, n, R\right)=-\ln \left\{\left(1-T_{0}\right)\left[\Gamma\left(r_{0}\right)+\sum_{i=1}^{n} \Gamma\left(r_{i 2}\right)-\sum_{i=1}^{n} \Gamma\left(r_{i 1}\right)\right] R\right\}
$$

Where

$$
\Gamma(x)=\sum_{m=0}^{l} \frac{1}{(l-m) !}\left(\frac{2 x^{2}}{\omega_{0}^{2}}\right)^{l-m} \exp \left(\frac{2 x^{2}}{\omega_{0}^{2}}\right)
$$

where $\omega_{0}$ is the radius of the laser fundamental mode $\left(\mathrm{TEM}_{00}\right)$ on OC. By calculating the integral in Eq. 1 and submit Eq. 2 into Eq. 1, the threshold pump power for LG $_{0 l}$ mode oscillation can be simplified as (Details in Supplementary Discussion I)

$$
P_{t h}\left(L G_{0 l}\right) \propto \frac{\left(1+\mu^{2}\right)^{l+1}}{\mu^{2 l}} L_{0}-\frac{\left(1+\mu^{2}\right)^{l+1}}{\mu^{2 l}} \ln \left\{\left(1-T_{0}\right)\left[\Gamma\left(r_{0}\right)+\sum_{i=1}^{n} \Gamma\left(r_{i 2}\right)-\sum_{i=1}^{n} \Gamma\left(r_{i 1}\right)\right] R\right\}
$$

where $\mu$ is the ratio of the pump beam radius to the laser fundamental mode radius in the gain crystal. By calculating Eq. 4, relationship between vortex oscillation pump threshold and the topological charge $l$ can be obtained, as shown in Fig. $2 \mathrm{~b}$. It is worth noting that, the diffraction loss in the laser is very small and is ignored in the calculation.

\section{Build-up of multi-vortex laser}

The multi-vortex laser is built as designed in Fig. 2a. In detail, the pump source is a fiber-coupling LD at $808 \mathrm{~nm}$, the focal length of the collimated lens L1 and the focal lens $\mathrm{L} 2$ are both $10 \mathrm{~cm}$, the gain crystal is a-cut $\mathrm{Nd}: \mathrm{YVO}_{4}$ crystal with the size of $3 \times$ $3 \times 3 \mathrm{~mm}^{3}$ and a doping concentration of $0.5 \%$, the curvature radius of M1 is -100 $\mathrm{mm}$, the transmittance of plano-plano $\mathrm{OC}$ is $2 \%$, and the cavity length is $4.5 \mathrm{~cm}$. The concentric-ring pattern is printed on OC using a high-energy Q-switched laser which is controlled by computer. In detail, the designed parameters of the concentric-ring pattern are input into computer, and the pattern is printed by the laser automatically. The Q-switched laser has an average output power of $300 \mathrm{~W}$ and a pulse repetition rate of $50 \mathrm{~Hz}$, and the center wavelength is $355 \mathrm{~nm}$. We print the pattern at Ronggao Laser Company (Shanghai, China). For multi-vortex beams generation, the center of the concentric-rings pattern is adjusted to the cavity axis. Meanwhile, the position of the focal lens L2 is adjusted to optimize the pump mode area matching with the area of the multi-vortex beams.

\section{Measurement of the topological charge}

In the experiment, ring-shaped apertures are used to separately select the multi-vortex beams to pass. Then, the selected vortex beam is focused into the central position of the cylindrical lens mode converter. The cylindrical lens mode converter is composed of two identical cylindrical lenses. The distance between the cylindrical lenses is $\sqrt{ } 2 f$, where $f$ is the focal length of the cylindrical lenses. Then the vortex beams are converted into HG beams. By counting the nodal line numbers of HG beams, the topological charges are obtained. 


\title{
Spatial encoding of multi-vortex beams
}

In the experiment, a DMD (V-7001, from VIALUX company, Germany) is used to realize the proposed hexadecimal spatial encoding. The DMD consists of micro-mirror arrays on the surface, and the angles of the micro-mirrors can be locally controlled by a computer. Hence, the reflection directions of incident beams can be locally controlled. According to the working principle of DMD, when the multi-vortex laser is incident onto the DMD, a specific combination of the vortex beams can be controlled to be reflected to the data transmission direction, then hexadecimal encoding of the multi-vortex laser is realized.

\section{Temporal encoding and TDM of pulsed vortex beams}

In the experiment, the pulsed $\mathrm{LG}_{01}$ beam is firstly divided into two beams using a splitter, then the two divided pulsed beams are fed into electro-optical modulators for temporal encoding. In the encoding process, binary signals are transferred into time-varying voltage signals that are synchronized with the pulses. Then, the voltage signals are loaded onto the electro-optic modulators, forming time-varying transmittance functions which are shown in the middle part of Fig. 6b. Finally, the pulsed beams are modulated and loaded with binary signals. Before multiplexing of the two temporally encoded pulsed beams, a delay line is used in one of the divided pulsed beams to introduce a half-pulse-cycle delay. Then multiplexing with no crosstalk is achieved by using a combiner.

\section{Supplementary information}

Supplementary information accompanies this paper at https://doi.org/10.1186/s43074-020-00013-X.

Additional file 1. Includes: Section 1. Derivation of relationship between vortex oscillation pump threshold and the concentric-rings pattern parameters; Section 2. Self-mode-locking results of $L_{G}, 118$ vortex beam; Section 3. Experiments of time-space division multiplexing of multi-vortex beams

\begin{abstract}
Abbreviations
OAM: Orbital angular momentum; SLM: Spatial light modulator; SPP: Spiral phase plate; LG: Laguerre-Gaussian; MOTMA: Multi-vortex oscillation threshold minimum analyzing; OC: Output coupler; LD: Laser diode; HG: HermiteGaussian; DMD: Digital micromirror device; TDM: Time-division multiplexing; SDM: Space-division multiplexing
\end{abstract}

Acknowledgements

Not applicable.

\section{Authors' contributions}

GX and ZQ conceived original idea of multi-vortex beams generation, JW conceived the ideal of spatial encoding and temporal encoding with multi-vortex laser. JW and ZW designed the experiments of vortex-enabled optical communications. ZQ performed the theoretical calculation and the multi-vortex generation experiment. ZQ and ZW performed the experiments of vortex communications with equivalent contributions. LQ and DF provided technical supports. All authors contributed to writing. GX. and JW. finalized the paper. GX and JW supervised the project. The author(s) read and approved the final manuscript.

Funding

The work is supported by the National Natural Science Foundation of China Grant No. 61675130, 11774116, 11721091, 61490713, 91850203, 61761130082, 11574001, the National Key R\&D Program of China (2018YFB2200204

2018YFB1801803), the Royal Society-Newton Advanced Fellowship, the Natural Science Foundation of Hubei Province of China (2018CFA048), the Key R\&D Program of Guangdong Province (2018B030325002), the Program for HUST Academic Frontier Youth Team (2016QYTD05), and the Fundamental Research Funds for the Central Universities (2019kfyRCPY037).

Availability of data and materials

The datasets used and/or analysed during the current study are available from the corresponding author on reasonable request. 


\section{Author details}

'School of Physics and Astronomy, Key Laboratory for Laser Plasmas (Ministry of Education), Collaborative Innovation center of IFSA (CICIFSA), Shanghai Jiao Tong University, Shanghai 200240, China. ${ }^{2}$ Wuhan National Laboratory for Optoelectronics and School of Optical and Electronic Information, Huazhong University of Science and Technology, Wuhan 430074, China. ${ }^{3}$ SZU-NUS Collaborative Innovation Center for Optoelectronic Science and Technology, and Key Laboratory of Optoelectronic Devices and Systems of Ministry of Education and Guangdong Province, College of Optoelectronic Engineering, Shenzhen University, Shenzhen 518060, China.

Received: 18 December 2019 Accepted: 6 February 2020

Published online: 15 May 2020

\section{References}

1. Yao A, Padgett MJ. Orbital angular momentum: origins, behavior and applications. Adv Opt Photon. 2011;3:161-204.

2. Franke-Arnold S, Allen L, Padgett MJ. Advances in optical angular momentum. Laser Photon Rev. 2008;2:299-313.

3. Dholakia K, Čižmár T. Shaping the future of manipulation. Nature Photon. 2002;5:335-42.

4. MacDonald MP, Paterson L, Volke-Sepulveda K, Arlt J, Sibbett W, Dholakia K. Creation and manipulation of threedimensional optically trapped structures. Science. 2002;296:1101-3.

5. Padgett MJ, Bowman R. Tweezers with a twist. Nature Photon. 2011;5:343-8.

6. Fürhapter S, Jesacher A, Bernet S, Ritsch-Marte M. Spiral phase contrast imaging in microscopy. Opt Express. 2005;13: 689-94.

7. Fang L, Padgett MJ, Wang J. Sharing a common origin between the rotational and linear Doppler effects. Laser \& Photon Rev. 2017;11:1700183.

8. Vieira J, Trines RMGM, Alves EP, Fonseca RA, Mendonca JT, Bingham R, et al. Amplification and generation of ultraintense twisted laser pulses via stimulated Raman scattering. Nat Commun. 2016;7:10371.

9. Elias NM. Photon orbital angular momentum in astronomy. Astron Astrophys. 2008;492:883-922.

10. Mair A, Vaziri A, Weihs G, Zeilinger A. Entanglement of the orbital angular momentum states of photons. Nature. 2001; 412:313-6.

11. Allen L, Beijersbergen MW, Spreeuw RJC, Woerdman JP. Orbital angular momentum of light and the transformation of Laguerre-Gaussian laser modes. Phys Rev A. 1992;45:8185-9.

12. Gibson G, Courtial J, Padgett MJ, Vasnetsov M, Pas'ko V, Barnet SM, et al. Free-space information transfer using light beams carrying orbital angular momentum. Opt Express. 2004;12:5448-56.

13. Wang J, Yang JY, Fazal IM, Ahmed N, Yan Y, Huang H, et al. Terabit free-space data transmission employing orbital angular momentum multiplexing. Nature Photon. 2012;6:488-96.

14. Lei T, Zhang M, Li Y, Jia P, Liu GN, Xu X, et al. Massive individual orbital angular momentum channels for multiplexing enabled by Dammann gratings. Light: Sci Appl. 2015;4:e257.

15. Wang J. Advances in communications using optical vortices. Photon Res. 2016;4:B14-28.

16. Wang J. Data information transfer using complex optical fields: a review and perspective. Chin Opt Lett. 2017;15:030005.

17. Wang J. Metasurfaces enabling structured light manipulation: advances and perspectives. Chin Opt Lett. 2018;16:050006.

18. Bozinovic N, Yue $Y$, Ren $Y$, Tur M, Kristensen $P$, Huang $H$, et al. Terabit-scale orbital angular momentum mode division multiplexing in fibers. Science. 2013;340:1545-8.

19. Wang A, Zhu L, Liu J, Du C, Mo Q, Wang J. Demonstration of hybrid orbital angular momentum multiplexing and timedivision multiplexing passive optical network. Opt Express. 2015;23:29457-66.

20. Wang A, Zhu L, Chen S, Du C, Mo Q, Wang J. Characterization of LDPC-coded orbital angular momentum modes transmission and multiplexing over a 50-km fiber. Opt Express. 2016;24:11716-26.

21. Chen S, Liu J, Zhao Y, Zhu L, Wang A, Li S, et al. Full-duplex bidirectional data transmission link using twisted lights multiplexing over 1.1-km orbital angular momentum fiber. Sci. Rep. 2016;6:38181.

22. Zhu L, Liu J, Mo Q, Du C, Wang J. Encoding/decoding using superpositions of spatial modes for image transfer in kmscale few-mode fiber. Opt Express. 2016;24:16934-44.

23. Zhu L, Wang A, Chen S, Liu J, Mo Q, Du C, et al. Orbital angular momentum mode groups multiplexing transmission over 2.6-km conventional multi-mode fiber. Opt. Express. 2017;25:25637-45.

24. Wang A, Zhu L, Wang L, Ai J, Chen S, Wang J. Directly using 8.8-km conventional multi-mode fiber for 6-mode orbital angular momentum multiplexing transmission. Opt. Express. 2018;26:10038-47.

25. Willner AE, Huang H, Yan Y, Ren Y, Ahmed N, Xie G, et al. Optical communications using orbital angular momentum beams. Adv Opt Photon. 2015;7:66-106.

26. Wang J. Twisted optical communications using orbital angular momentum. Sci China Phys Mech Astron. 2019;62: 034201.

27. Liu J, Li SM, Zhu L, Wang AD, Chen S, Klitis C, et al. Direct fiber vector eigenmode multiplexing transmission seeded by integrated optical vortex emitters. Light: Sci Appl. 2018;7:17148.

28. Fu S, Zhai Y, Zhou H, Zhang J, Wang T, Yin C, et al. Demonstration of free-space one-to many multicasting link from orbital angular momentum encoding. Opt Lett. 2019:44:4753-6.

29. Fu S, Zhai Y, Zhou H, Zhang J, Wang T, Liu X, et al. Experimental demonstration of free-space multi-state orbital angular momentum shift keying. Opt Express. 2019;27:33111-9.

30. Forbes A, Dudley A, McLaren M. Creation and detection of optical modes with spatial light modulators. Adv Opt Photon. 2016:8:200-27.

31. Beijersbergen MW, Coerwinkel RPC, Kristensen M, Woerdman JP. Helical wavefront laser beams produced with a spiral phase plate. Opt Commun. 1994;112:321-7.

32. Zhu L, Wang J. Simultaneous generation of multiple orbital angular momentum (OAM) modes using a single phaseonly element. Opt Express. 2015;23:26221-33.

33. Li S, Wang J. Adaptive power-controllable orbital angular momentum (OAM) multicasting. Sci Rep. 2015;5:9677.

34. Kim DJ, Kim JW. Direct generation of an optical vortex beam in a single-frequency Nd: YVO4 laser. Opt Lett. 2015:40: 399-402. 
35. Liu Q, Zhao Y, Zhou W, Zhang J, Wang L, Yao W. Shen D (2017) control of vortex helicity with a quater-wave plate in an Er: YAG ceramic solid state laser. IEEE Photonics J. 2017;9:1500408.

36. Huang $X, X u B, C$ Cui $S, X u H, C$ Cai Z, Chen L. Direct generation of vortex laser by rotating induced off-axis pumping. IEEE J Sel Top Quantum Electron. 2018;24:1601606.

37. Wang S, Zhang SL, Qiao HC, Li P, Hao MH, Yang HM, et al. Direct generation of vortex beams from a double-end polarized pumped Yb: KYW laser. Opt Express. 2018;26:26925-32

38. Ito A, Kozawa Y, Sato S. Generation of hollow scalar and vector beams using a spot-defect mirror. J Opt Soc Am A. 2010;27:2072-7.

39. Zhou J, Zhang W, Chen L. Experimental detection of high-order or fractional orbital angular momentum of light based on a robust mode converter. Appl Phys Lett. 2016;108:111108.

40. Beijersbergen MW, Allen L, Van Der Veen HELO, Woerdman JP. Astigmatic laser mode converters and transfer of orbital angular momentum. Opt Commun. 1993;96:123-32.

41. Zhang Y, Yu H, Zhang H, Xu X, Xu J, Wang J. Self-mode-locked Laguerre-Gaussian beam with staged topological charge by thermal-optical field coupling. Opt Express. 2016;24:5514-22.

42. Chang MT, Liang HC, Su KW, Chen YF. Exploring transverse pattern formation in a dual-polarization self-mode-locked monolithic Yb: KGW laser and generating a 25-GHz subpicosecond vortex beam via gain competition. Opt Express. 2016;24:8754-62.

43. Li Z, Peng J, Li Q, Gao Y, Li J, Cao Q. Generation of picosecond vortex beam in a self-mode-locked Nd:YVO 4 laser. Opt Lett. 2017;13:188-91.

44. Liang HC, Huang YJ, Lin YC, Lu TH, Chen YF, Huang KF. Picosecond optical vortex converted from multigigahertz selfmode-locked high-order Hermite-Gaussian Nd: GdVO4 lasers. Opt Lett. 2009;34:3842-4.

45. Igarashi K, Katoh K, Kikuchi K, Imai K, Kourogi M. Generation of 10-GHz 2-ps optical pulse train over the $\mathrm{C}$ band based on an optical comb generator and its application to 160-Gbit/s OTDM systems. In: 34th European conference on optical communication paper Tu. 3. D. I; 2008.

46. Hu H, Mulvad HCH, Peucheret C, Galili M, Clausen A, Jeppesen P, et al. $10 \mathrm{GHz}$ pulse source for $640 \mathrm{Gbit} / \mathrm{s}$ OTDM based on phase modulator and self-phase modulation. Opt Express. 2011;19:343-9.

47. Chen YF, Huang YJ, Chiang PY, Lin YC. Controlling number of lasing modes for designing short-cavity self-mode-locked Nd-doped vanadate lasers. Appl Phys B Lasers Opt. 2010;103:841-6.

48. Huang YJ, Tzeng YS, Cho HH, Chen YF. Effect of spatial hole burning on a dual-wavelength mode-locked laser based on compactly combined dual gain media. Photon.Res. 2014;2:161-7.

49. Bai Y, Chen S, Wang Z, Zhang G. Novel self-mode-locking mechanism in narrow-band lasers. Appl Phys Lett. 1993;63: 2597-9.

50. Shi JY, Fang Y, Chi N. Time division multiplexed orbital angular momentum access system. Optim Eng. 2016;55:036106.

51. Chen YF, Chang MY, Zhuang WZ, Su KW, Huang KF, Liang HC. Generation of sub-terahertz repetition rates from a monolithic self-mode-locked laser coupled with an external Fabry-Perot cavity. Laser Photon Rev. 2015;9:91-7.

52. Clarkson WA, Hanna DC. Effects of transverse-mode profile on slope efficiency and relaxation oscillations in a longitudinally-pumped laser. J Mod Opt. 1989;36:483-98.

\section{Publisher's Note}

Springer Nature remains neutral with regard to jurisdictional claims in published maps and institutional affiliations.

\section{Submit your manuscript to a SpringerOpen ${ }^{\circ}$ journal and benefit from:}

- Convenient online submission

- Rigorous peer review

- Open access: articles freely available online

- High visibility within the field

- Retaining the copyright to your article

Submit your next manuscript at $\boldsymbol{s p r i n g e r o p e n . c o m ~}$ 